Research Paper

\title{
Knockdown of Tubulin Polymerization Promoting Protein Family Member 3 inhibits cell proliferation and invasion in human colorectal cancer
}

\author{
Kuanping $\mathrm{Ye}^{1^{*}}$, Yintao $\mathrm{Li}^{2}{ }^{3}{ }^{*}$, Weiwei Zhao ${ }^{4}$, Nan $\mathrm{Wu}^{5}$, Naijia Liu ${ }^{1}$, Rumei Li ${ }^{1}$, Lili Chen ${ }^{1}$, Min $\mathrm{He}^{1}$, Bin Lu${ }^{1}$, \\ Xuanchun Wang ${ }^{1}$, Renming $\mathrm{Hu}^{1 凶}$ \\ 1. Department of Endocrinology and Metabolism, Institute of Endocrinology and Diabetology, Huashan Hospital, Fudan University, Shanghai, P.R. China \\ 2. Department of Medical Oncology, Shandong Cancer Hospital and Institute, Jinan, Shandong, P.R. China \\ 3. School of Medicine, Shandong University, Jinan, P.R. China \\ 4. Department of Integrated Therapy, Fudan University Shanghai Cancer Center, Shanghai, P.R. China \\ 5. Department of Geriatrics, Zhongshan Hospital, Fudan University, Shanghai, P.R. China \\ *These authors contributed equally to this work. \\ $\triangle$ Corresponding author: Renming Hu, MD, Email: renminghu@fudan.edu.cn \\ ( $)$ Ivyspring International Publisher. This is an open access article distributed under the terms of the Creative Commons Attribution (CC BY-NC) license \\ (https://creativecommons.org/licenses/by-nc/4.0/). See http://ivyspring.com/terms for full terms and conditions.
}

Received: 2016.12.27; Accepted: 2017.04.25; Published: 2017.07.01

\begin{abstract}
Tubulin Polymerization Promoting Protein Family Member 3 (TPPP3), a member of the TPPP protein family, has been reported to play important roles in initiation and progression of human cancers. However, the expression and underlying function of TPPP3 in colorectal cancer (CRC) have not yet been fully clarified. In this study, the mRNA and protein levels of TPPP3 in 96 clinical CRC specimens were determined by RT-PCR and immunohistochemistry. The relation between TPPP 3 expression and clinicopathologic characteristics and overall survival (OS) were evaluated. Further experiments showed that knockdown of TPPP3 inhibited cell proliferation, migration and invasion and induced cell apoptosis in vitro. In addition, TPPP3 silencing resulted in a decrease of angiogenesis and S phase fraction. Thus, our results suggested that TPPP3 played an important role in CRC progress and might serve as novel therapeutic target for CRC treatment.
\end{abstract}

Key words: colorectal cancer, TPPP3, proliferation, invasion, apoptosis

\section{Introduction}

Colorectal cancer (CRC) is one of the most common types of malignant tumor and the third leading cause of cancer-related deaths in the world [1, 2]. The occurrence rate is significantly increasing and CRC has become the fifth most frequent cause of cancer-related death in China [3]. Despite significant improvement in curative surgical resection and adjuvant chemotherapeutic regimens, the majority of patients are often diagnosed at an advanced stage thus with poor prognosis [4-6]. A growing number of studies have demonstrated that complicated pathogenesis of CRC was a multi-step process involving in the deregulation of multiple oncogenes and tumor suppressors [7-10], thereby emphasizing the need to further understand the molecular mechanisms underlying the formation and development of CRC.

TPPP3, a member of the tubulin polymerization-promoting protein family, was reported to induce tubulin polymerization and microtubule bundling both in vitro and in vivo [11]. TPPP3 was reported to express in joints coincides and represent a molecular marker in joint differentiation [12]. We previously reported that depletion of TPPP3 suppressed cell proliferation and induced cell cycle arrest by regulating centrosomes amplification and/or spindles translocation processes in HeLa cells [13]. Furthermore, TPPP3 was found to increase in NSCLC tissues and associate with short overall survival. Knockdown of TPPP3 inhibited cell 
proliferation and induced cell apoptosis and cell cycle arrest in vitro and suppressed tumor growth in vivo $[14,15]$. Together with those findings, it seems likely that TPPP3 played as an oncogene in cancer.

In the present study, we further assessed whether TPPP3 played any role in tumor development and progress in CRC. Our result showed that TPPP3 was significantly increased in CRC tissues and associated with aggressive factors and poor patient survival. Moreover, we examined the biological function of TPPP3 in CRC cells in vitro.

\section{Materials and Methods}

\section{Clinical samples}

A total of 96 human primary CRC tissues and their adjacent noncancerous tissues were collected from patients who had undergone gastrointestinal surgery between 2008 and 2010 at Huashan Hospital of Fudan University, China. None of the patients had received radiotherapy, chemotherapy, or other treatments before surgery. Written informed consent was obtained from all enrolled patients, and all investigations were performed according to the declaration of Helsinki. This study was approved by the Ethics Committee of Huashan Hospital of Fudan University, Shanghai, China.

\section{Cell lines and cultivation}

Human CRC cell lines SW480, HT29, RKO, LOVO, and SW620, and the normal human intestinal epithelial cell line HIEC were obtained from cell banks of the Shanghai Institutes of Biological Sciences (Shanghai, China). All cells were cultured in RPMI-1640 (Gibco, Grand Island, NY, USA) medium supplemented with 10\% fetal bovine serum (Gibco), 1 $\%$ streptomycin/penicillin in a humidified tissue culture incubator at $37^{\circ} \mathrm{C}$ in $5 \% \mathrm{CO} 2$.

\section{Quantitative real-time PCR analysis}

Total RNA was isolated from clinical specimen and CRC cell lines using TRIzol ${ }^{\circledR}$ Reagent (Life Technologies, USA) according to the manufacturer's instructions. Complementary DNA synthesis and the quantitative real-time PCR were performed as our previously reported [15]. The level of TPPP3 was expressed as a ratio relative to the GAPDH.

\section{Immunohistochemistry (IHC)}

The protein expression of TPPP3 was measured by IHC method that was performed on $4 \mu \mathrm{m}$ paraffin-embedded CRC tissue sections as our previously reported [15]. In brief, slides were deparaffinized in xylene, rehydrated using graded alcohol, pre-treated with $10 \mathrm{mM}$ citrate buffer $\mathrm{pH}=6$ and blocked for endogenous peroxidase activity in 3
$\% \mathrm{H}_{2} \mathrm{O}_{2}$. The slides were incubated with primary antibody against TPPP3 (ABGENT, China), followed by horseradish peroxidase-linked secondary antibody (DAKO, Denmark, CA, USA) for 30 minutes at room temperature. The slides were counterstained with Mayer's hematoxylin, dehydrated and mounted. The TPPP3 protein expressions were scored by multiplying the proportion of positive cells and intensity using the semiquantitative scale described previously [15].

\section{Plasmid and lentivirus infection}

Stable knock down of TPPP3 was performed using short hairpin RNA (shRNA) lentiviral constructs as we described previously [15]. CRC cells were infected with scramble sequence (SCR) or sequences targeting TPPP3 for $24 \mathrm{~h}$. Following this step, the expression levels of TPPP3 were measured by Western blotting.

\section{Cell proliferation and clone formation assay}

Cell proliferation and clone formation were performed as we previously reported [15].

\section{Cell apoptosis and cell cycle analysis assay}

Cell apoptosis and cell cycle were performed using flow cytometry as we previously reported [15].

\section{Cell migration and invasion assay}

Cell migration assays were performed using 24-well Transwell units with $8 \mu \mathrm{m}$ pore size polycarbonate inserts (Corning, NY, USA). About $4 \times$ $10^{4}$ cells were harvested in $200 \mu \mathrm{L}$ serum-free medium and seeded into the upper chamber. $600 \mu \mathrm{L}$ medium containing $10 \%$ fetal bovine serum was placed in the lower chamber. After $36 \mathrm{~h}$ incubation, cells remaining on the upper surface of the insert membrane were wiped off with a cotton swab, and cells through the insert membrane were stained with $0.5 \%$ crystal violet and counted in five random fields under 100x magnification. Representative images of migrated cells were captured under a microscope (Olympus, Tokyo, Japan).

Invasion assay was performed as the same procedure with the polycarbonate inserts precoated with Matrigel (BD Biosciences, San Jose, CA, USA).

\section{HUVEC tube formation assay}

HUVECs $\left(4 \times 10^{4} /\right.$ well $)$ in different conditioned media from CRC cells infected with SCR or shRNA targeting TPPP3 viral were seeded in 96-well plates coated with $200 \mu \mathrm{L}$ Matrigel. The 24-well plates were incubated for $6 \mathrm{~h}$, and tube formation was then photographed in five random fields under an inverted microscope. The endothelial tubes were quantified by counting length and branches per image field using 
NIH Image J software.

\section{Western blot analysis}

For the protein expression analyses, standard Western blot assay was performed. Cells were lysed in RIPA buffer (Cell Signaling Technology, Danvers, MA, USA), and the cell lysates were separated by 10 $\%$ SDS-PAGE and transferred to PVDF membranes (Bio-Rad, CA, USA). After blocking with $5 \%$ non-fat milk, membranes were incubated with BCL-2, Bax, STAT3, p-STAT3 (ser727), VEGF, P21, MMP-9, E-cadherin and Rac-1 (all from Cell Signaling Technology) antibodies overnight at $4^{\circ} \mathrm{C}$. Following by incubation with the appropriate HRP-conjugated secondary antibody for $1 \mathrm{~h}$ at room temperature, membranes were detected using SuperSignal West Dura Extended Duration Substrate (Pierce, Rockford, IL, USA).

\section{Statistical analysis}

Data were expressed as mean \pm standard deviation (SD) of three independent experiments. Data of in-vitro experiments were analyzed using Student's t-test or

One-Way ANOVA. Pearson Chi-square test was performed to assess the relationship between TPPP3 expression and clinic-pathological features. Survival curves were calculated by the Kaplan-Meier method, and the statistical analyses were analysed using the log-rank test. P-values less than 0.05 were considered statistically significant.

\section{Results}

\section{TPPP3 expression is upregulated in human CRC tissues and cell lines}

To investigate the roles of TPPP3 in human CRC,


Figure 1. TPPP3 expression is increased in CRC tissues and cells. (A) The mRNA expression of TPPP3 was measured by qRT-PCR in 96 pairs of human CRC tissues (CRC) and adjacent non-tumorous tissues. (B) qRT-PCR was performed to detect TPPP3 mRNA expression in 5 CRC cell lines and the normal intestinal epithelial cell line HIEC. (C) Detection of TPPP3 protein expression by Western blotting in CRC cell lines and HIEC. The expression levels of TPPP3 were normalized with GAPDH. $* * \mathrm{P}<0.01$. 
A

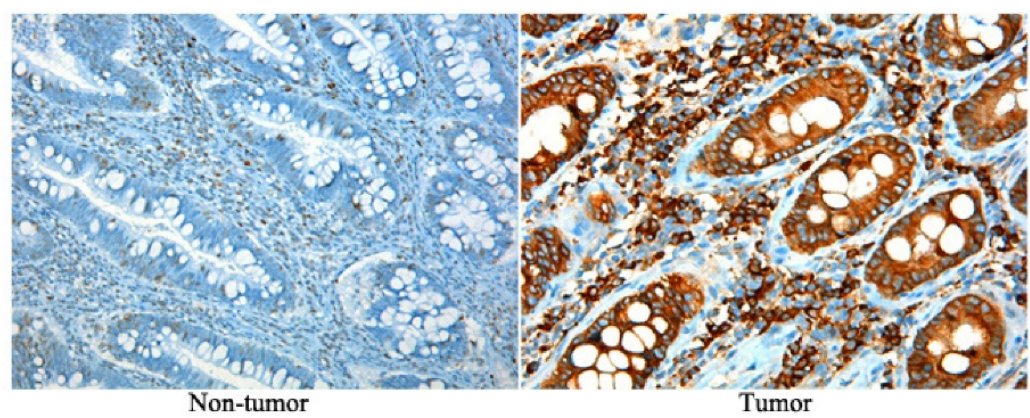

B

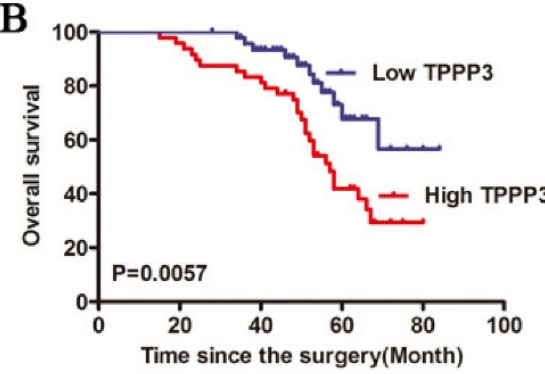

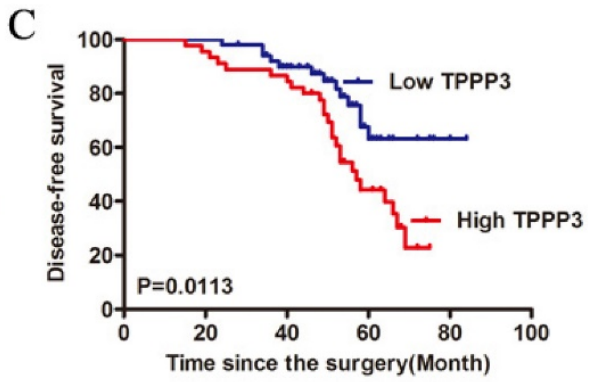

Figure 2. High TPPP3 expression was associated with progression and poor prognosis in CRC. (A) Representative images of TPPP3 expression in adjacent non-cancerous tissue and CRC specimens examined by IHC analysis. (B) Kaplan-Meier overall survival curves for 96 patients with CRC according to TPPP3 expression. The median TPPP3 expression level in the tumor samples was chosen as the cut-off point. (C) DFS curves of CRC patients with high TPPP3 expression low TPPP3 expression.

Table 1. Relationship between clinicopathological factors and TPPP3 expression in primary CRC

\begin{tabular}{llll}
\hline \multicolumn{4}{l}{ TPPP3 expression } \\
\hline Variable & High $(\mathrm{n}=48)$ & Low $(\mathrm{n}=48)$ & P-value \\
\hline Age (years) & 20 & 26 & 0.3070 \\
$\leq 50$ & 28 & 22 & \\
$>50$ & & & 0.6780 \\
Gender & 27 & 30 & \\
Male & 21 & 18 & \\
Female & & & 0.0038 \\
Tumor size & 14 & 29 & \\
$\leq 5 \mathrm{~cm}$ & 34 & 19 & \\
$>5 \mathrm{~cm}$ & & & \\
Histological grading & 28 & 31 & \\
Well, moderate & 20 & 17 & \\
Poor & & & \\
TNM stage & 17 & 34 & \\
Stage I/II & 31 & 14 & \\
Stage III/IV & & & \\
Location & 29 & 31 & \\
Colon & 19 & 17 & \\
Rectum & & & \\
Lymph node metastasis & 19 & 33 & \\
No & 29 & 15 & \\
Yes &
\end{tabular}

Well: well-differentiated, moderate: moderately differentiated, poor: poorly differentiated, TNM: tumor-node-metastasis.

\section{Knockdown of TPPP3 suppresses CRC cell proliferation in vitro}

In order to explore the possible role of TPPP3 in the development and progression of CRC, stable knockdown TPPP3 expression in LOVO and SW620 cell lines, which had higher endogenous TPPP3 expression, was made. Knockdown of TPPP3 significantly decreased the expression of TPPP3 compared with SCR both in LOVO and SW620 cells (Figure 3A). shRNA2 targeting TPPP3 was the most highly functional shRNA and was used in the following experiments. The proliferation of both cells was measured using CCK- 8 assay, which revealed that depletion of TPPP3 caused a significant decrease in proliferation of LOVO and SW620 (Figure 3B). Colony formation assay suggested that downregulation of TPPP3 significantly inhibited the proliferation rate of LOVO and SW620 cells compared to SCR (Figure 3C). In cells infected with shTPPP3, the apoptotic ratio of cells was markedly increased compared with cells infected with scrambled shRNA (Figure 3D). Correspondingly, apoptotic protein markers, as characterized by an increase in Bax and a decrease in the levels of the antiapoptotic proteins BCL-2 (Figure 3E). The p-STAT3 (ser727) expression was significantly decreased in LOVO and SW620 cells infection with TPPP3 shRNA (Figure 3E). Our results demonstrated that downregulation of TPPP3 inhibited oncogenic growth and proliferation in vitro. 
A


$\mathrm{B}$
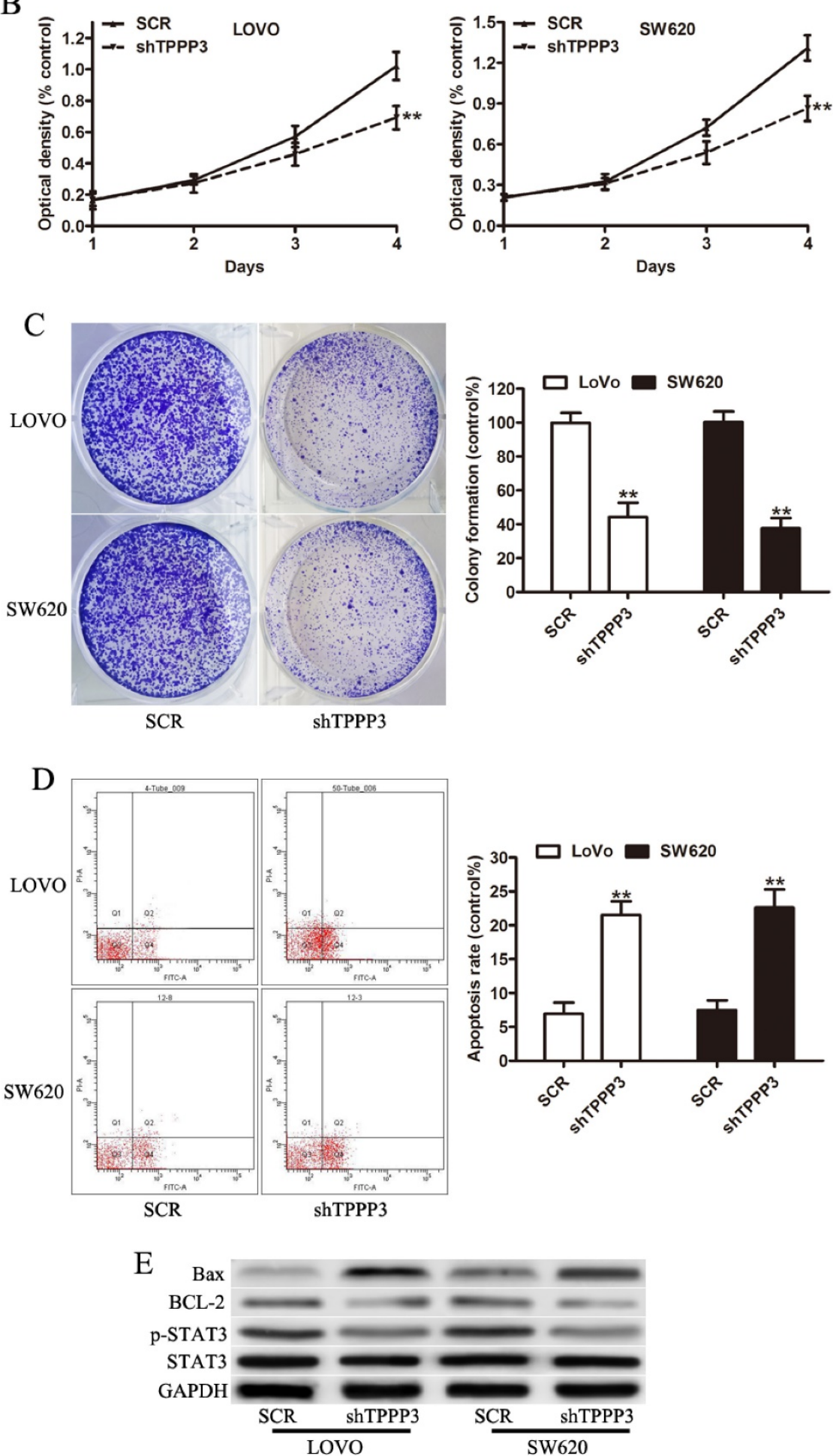

Figure 3. Knockdown of TPPP3 inhibited the proliferation of CRC cells in vitro. (A) Expression of TPPP3 in stable knockdown was determined by Western blot analysis in LOVO and SW620 cells. (B) Cell proliferation was determined by CCK-8 in LOVO and SW620 cells infected with TPPP3 shRNA or SCR. (C) Representative micrographs of colony formation of CRC cells infected with TPPP3 shRNA or SCR were shown. (D) CRC cells infected with TPPP3 shRNA or SCR were stained with PI and Annexin-FITC for the evaluation of apoptosis by flow cytometric analysis. (E) Western blot analysis of Bax, BCL-2, P-STAT3 and STAT3 expression in LOVO and SW620 cells infected with TPPP3 shRNA or SCR. 


\section{TPPP3 silencing inhibits angiogenesis and decreases $S$ phase fraction in CRC cells}

Angiogenesis has been shown to be frequently altered in tumor progression [16-18]. Then we determined the effect of conditioned media from CRC cells on HUVEC cells. Media from TPPP3 knockdown LOVO and SW620 cells resulted in a significant reduction of the number of tuber structures compared to media of SCR cells (Figure 4A). Cell cycle analysis showed that the percentage of cells in $S$ phase decreased while the percentage of cells in G1 phase increased after TPPP3 knockdown in LOVO cells (Fig 4B). Cell cycle analysis showed that the percentage of cells in S phase decreased while the percentage of cells in G2/M phase increased after TPPP3 knockdown in SW620 cells (Fig 4B). These data indicated that TPPP3 knockdown prevented the CRC cells from entering the mitotic phase. To assess whether TPPP3 could regulate the genes involved in angiogenesis and cell-cycle, we measured the expression of VEGF and P21. Western blotting indicated that TPPP3 silencing decreased the expression of VEGF and P21 in both LOVO and SW620 cells (Fig 4C). Taken together, these data indicated that TPPP3 might regulate angiogenesis and cell cycle through the expression of VEGF and P21 in CRC cells.


Figure 4. Effect of TPPP3 on the angiogenesis and cell cycle of CRC cells. (A) Representative images of capillary-like structure stimulated by conditioned medium were shown. In vitro angiogenesis was quantitated as described in Materials and Methods. (B) Cell cycle distribution was determined by flow cytometric analysis. Bar graph represents the percentage distribution of CRC cells in different phases of cell cycle. (C) VEGF and P21 expression in CRC cells infected with TPPP3 shRNA or SCR were analyzed by Western blot. $* * P<0.01$. 

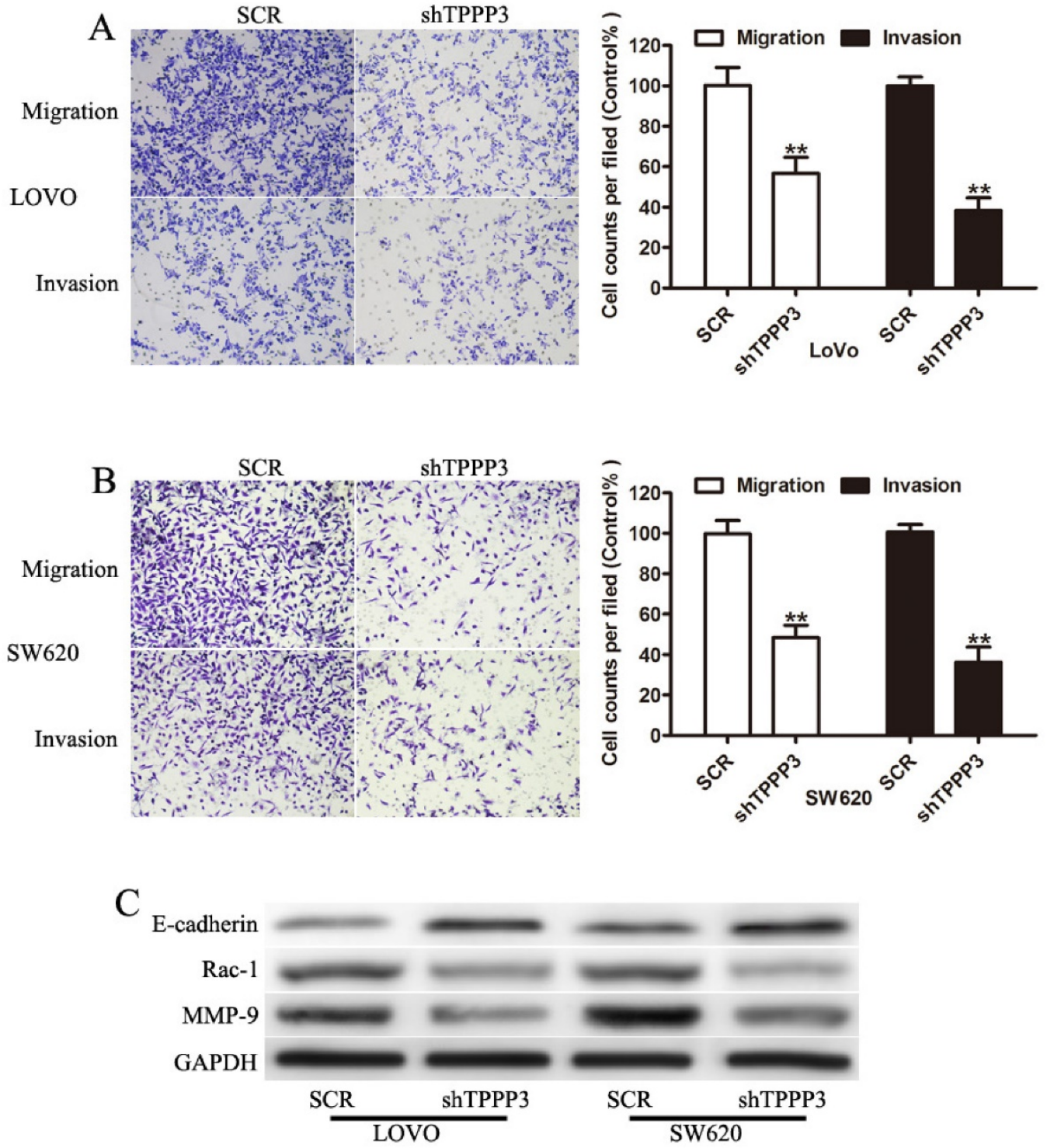

Figure 5. TPPP3 knockdown inhibited the cellular migration and invasion of CRC cells. (A) Representative field of migration (top) or invasive (bottom) LOVO cells on membrane. Average number of invasive or migration cells number per field from three independent experiments \pm SD. (B) Representative field of migration (top) or invasive (bottom) SW620 cells on membrane. Average number of invasive or migration cells number per field from three independent experiments \pm SD. (C) Expression of MMP-9, Rac-1, and E-cadherin were determined by Western blotting in LOVO and SW620 cells infected with TPPP3 shRNA or SCR. **P<0.01.

\section{Knockdown of TPPP3 impairs migration and invasion of CRC cells in vitro}

To further determined whether ТРPP3 affected migration and invasion of CRC cells, transwell assay was performed. Cells infected with TPPP3 shRNA showed a significant reduction in cell migration and invasion compared to SCR-transfection cells (Figure 4A and 4B). To evaluate whether TPPP3 has influence on the metastasis-related protein in CRC cells, Western blotting was carried out to detect the expression of MMP-9, Rac-1, and E-cadherin. Our results showed that knockdown of TPPP3 in LOVO and SW620 cells significantly decreased the expression of MMP-9 and Rac-1, while increased the expression of E-cadherin (Figure 5C). These results indicated that TPPP3 significantly affected the invasion and migration of CRC cells via the expression of MMP-9, Rac-1 and E-cadherin.

\section{Discussion}

Increasing studies have suggested that TPPP3 might be involved in malignant progression of several human cancers, including cervical cancer and non-small-cell lung cancer [13-15]. However, little is known about its role in CRC. In this study, we presented the first evidence that TPPP3 was significantly up-regulated in CRC tissues at both mRNA and protein levels as compared with adjacent 
non-cancerous tissues, suggesting a potential promotion of TPPP3 in colorectal tumor formation. Further investigation found that high expression of TPPP3 protein was significantly associated with aggressive characteristic and poor prognosis of patients. In accordance with our study, TPPP3 was reported to be associated with prognosis-related clinical parameters, including tumor size, lymph node metastasis and advanced clinical TNM stage [15]. Kaplan-Meier method showed overall survival rate was significantly lower in patients with high expression of TPPP3 than those with low expression of TPPP3. In addition, the DFS times of CRC patients with high expression of TPPP3 were significantly shorter than those of patients with low expression. These data suggested that TPPP3 might act as an oncogene in the tumor development and progress.

Then we explored the functions of TPPP3 in CRC cells. In vitro experiments revealed that silencing of TPPP3 in LOVO and SW620 cells with higher endogenous expression of TPPP3 could suppress cellular proliferation and induced cell apoptosis. Bax, BCL-2, and p-STAT3 have been reported to involve in cell proliferation and apoptosis in CRC [19-21]. At the molecular level, we observed an obvious decrease in the expression of BCL-2 and p-STAT3 and an increase of Bax expression. This is in line with our previous report showing TPPP3 silencing resulted in a decrease of BCL-2 and p-STAT3 expression [15]. It has been broadly accepted that metastasis was one of the most aggressive factors influencing patients' prognosis and relapse [22, 23]. Interestingly, we found knockdown of TPPP3 inhibited cell migration and invasion and positively correlated with MMP-9 and Rac-1 expression but negatively correlated with E-cadherin expression, suggesting a potential involvement of TPPP3 in metastasis phenotype. In accordance with our study, TPPP3 was reported to be one of the molecules essential for lung cell migration in vitro [14, 15]. In addition, we found that TPPP3 knockdown inhibited angiogenesis and led to a decrease of $S$ phase, accompanied with the downregulation of VEGF and P21 expression. Consistent with our previous report, TPPP3 silencing resulted in a decrease of $S$ phase in NSCLC cells [15]. Taken together, these findings provided the evidence that TPPP3 might be involved in development and progress of CRC. However, the specific regulatory role of TPPP3 remains to be necessary in CRC.

In conclusion, our study for the first time showed that TPPP3 was significantly upregulated in CRC and was associated with the progression and prognosis of CRC. Furthermore, TPPP3 knockdown inhibited CRC cell proliferation, migration, and invasion in vitro. These evidences suggested that
TPPP3 might be a potential therapeutic target for CRC patients in the forthcoming future.

\section{Acknowledgements}

This study was supported by the National Natural Science Foundation of China (No. 81471057, 81402288), Promotive research foundation for excellent young and middle-aged scientists of Shandong Province (No.BS2015YY028) and General Financial Grant from the China Postdoctoral Science Foundation (No.2016M602150).

\section{Competing Interests}

The authors have declared that no competing interests exist.

\section{References}

1. Siegel R, Desantis C, Jemal A. Colorectal cancer statistics, 2014. CA Cancer J Clin 2014,64:104-117.

2. Starlinger P, Herberger B, Tamandl D, Stremitzer S, Brostjan C, Gruenberger B, Gruenberger T. Low preoperative platelets to predict postoperative liver dysfunction in patients with metastatic colorectal cancer undergoing liver resection. J Clin Oncol 2012,30:634.

3. Siegel R, Naishadham D, Jemal A. Cancer statistics, 2012. CA Cancer J Clin 2012,62:10-29.

4. Hu L, Wang RY, Cai J, Feng D, Yang GZ, Xu QG, et al. Overexpression of CHKA contributes to tumor progression and metastasis and predicts poor prognosis in colorectal carcinoma. Oncotarget 2016.

5. Iinuma $\mathrm{H}$, Watanabe $\mathrm{T}$, Mimori $\mathrm{K}$, Adachi M, Hayashi N, Tamura J, et al. Clinical significance of circulating tumor cells, including cancer stem-like cells, in peripheral blood for recurrence and prognosis in patients with Dukes' stage B and C colorectal cancer. J Clin Oncol 2011,29:1547-1555.

6. Jawed I, Wilkerson J, Duffy AG, Fojo AT. Twenty years of chemotherapy for colorectal cancer (CRC): Progress made, insights gleaned. J Clin Oncol 2012,30:596

7. Fu T, Liu Y, Li K, Wan W, Pappou EP, Iacobuzio-Donahue CA, et al. Tumors with unmethylated MLH1 and the CpG island methylator phenotype are associated with a poor prognosis in stage II colorectal cancer patients. Oncotarget 2016

8. De Robertis M, Loiacono $\mathrm{L}$, Fusilli C Poeta ML, Mazza $\mathrm{T}$, Sanchez M, et al. Dysregulation of EGFR Pathway in EphA2 Cell Subpopulation Significantly Associates with Poor Prognosis in Colorectal Cancer. Clin Cancer Res 2016.

9. Yin C, Ma G, Rong Y, Kong P, Yang Q, Jiang C, et al. The Efficacy of Bevacizumab in Different Line Chemotherapy for Chinese Patients with Metastatic Colorectal Cancer. J Cancer 2016,7:1901-1906.

10. Figueroa-Gonzalez G, Garcia-Castillo V, Coronel-Hernandez J, Lopez-Urrutia E, Leon-Cabrera S, Arias-Romero LE, et al. Anti-inflammatory and Antitumor Activity of a Triple Therapy for a Colitis-Related Colorectal Cancer. J Cancer 2016,7:1632-1644.

11. Vincze O, Tokesi N, Olah J, Hlavanda E, Zotter A, Horvath I, et al. Tubulin polymerization promoting proteins (TPPPs): members of a new family with distinct structures and functions. Biochemistry-US 2006,45:13818-13826.

12. Staverosky JA, Pryce BA, Watson SS, Schweitzer R. Tubulin polymerization-promoting protein family member 3, Tppp3, is a specific marker of the differentiating tendon sheath and synovial joints. Dev Dyn 2009,238:685-692.

13. Zhou W, Wang X, Li L, Feng X, Yang Z, Zhang W, Hu R. Depletion of tubulin polymerization promoting protein family member 3 suppresses HeLa cell proliferation. Mol Cell Biochem 2010,333:91-98.

14. Zhou W, Li J, Wang X, Hu R. Stable knockdown of TPPP3 by RNA interference in Lewis lung carcinoma cell inhibits tumor growth and metastasis. Mol Cell Biochem 2010,343:231-238.

15. Li Y, Xu Y, Ye K, Wu N, Li J, Liu N, et al. Knockdown of Tubulin Polymerization Promoting Protein Family Member 3 Suppresses Proliferation and Induces Apoptosis in Non-Small-Cell Lung Cancer. J Cancer 2016,7:1189-1196.

16. Flores-Perez A, Marchat LA, Rodriguez-Cuevas S, Bautista-Pina V, Hidalgo-Miranda A, Ocampo EA, et al. Dual targeting of ANGPT1 and TGFBR2 genes by miR-204 controls angiogenesis in breast cancer. Sci Rep 2016,6:34504.

17. Khalid EB, Ayman EE, Rahman H, Abdelkarim G, Najda A. Natural products against cancer angiogenesis. Tumour Biol 2016,37:14513-14536.

18. Chen YS, Meng F, Li HL, Liu QH, Hou PF, Bai J, Zheng JN. Dicer suppresses MMP-2-mediated invasion and VEGFA-induced angiogenesis and serves as a 
promising prognostic biomarker in human clear cell renal cell carcinoma. Oncotarget 2016.

19. Ray P, Guha D, Chakraborty J, Banerjee S, Adhikary A, Chakraborty S, et al.

Crocetin exploits p53-induced death domain (PIDD) and FAS-associated death domain (FADD) proteins to induce apoptosis in colorectal cancer. Sci Rep 2016,6:32979.

20. Zhu DJ, Huang YF, Chen XW, Luo ZT, Wang GX, Liu CC, et al. Curcumin partly ameliorates irinotecan-induced diarrhea and synergistically promotes apoptosis in colorectal cancer through mediating oxidative stress. Oncotarget 2016.

21. Zhu X, Li D, Yu F, Jia C, Xie J, Ma Y, et al. miR-194 inhibits the proliferation, invasion, migration, and enhances the chemosensitivity of non-small cell lung cancer cells by targeting forkhead box A1 protein. Oncotarget 2016,7:13139-13152.

22. Labianca R, Beretta GD, Mosconi S, Pessi MA, Milesi L. The development of clinical research in CRC. Ann Oncol 2005,16 Suppl 4:v37-v43.

23. Feng $\mathrm{M}$, Feng J, Chen $\mathrm{W}$, Wang $\mathrm{W}$, Wu X, Zhang J, et al. Lipocalin2 suppresses metastasis of colorectal cancer by attenuating NF-kappaB-dependent activation of snail and epithelial mesenchymal transition. Mol Cancer 2016,15:77. 